\title{
Effect of Peripheral Arterial Disease on Serum Ischemia-Modified Albumin Levels in Type 2 Diabetic Patients
}

\author{
Tugra Gencpinar ${ }^{1}$, Umut Ayoğlu' ${ }^{1}$, Koray Aykut ${ }^{2}$, Gokhan Albayrak², Hamid Yaşar Ellidag3, \\ Muzaffer Yılmaz' ${ }^{1}$, Kadir Sağdıç ${ }^{1}$, Mustafa Emmiler ${ }^{1}$ \\ ${ }^{1}$ Cardiovascular Surgery Clinic, Education and Research Hospital, Antalya, Turkey \\ ${ }^{2}$ Department of Cardiovascular Surgery, Izmir University, Izmir, Turkey \\ ${ }^{3}$ Department of Biochemistry, Education and Research Hospital, Antalya, Turkey \\ Email: "tugra01@yahoo.com, drraifumut@hotmail.com, drkaykut@hotmail.com, albayrakg@hotmail.com, \\ hayael1980@hotmail.com, savan64@hotmail.com, sagdick@tnn.net, dremmiler@yahoo.com
}

Received 1 April 2014; revised 1 May 2014; accepted 8 May 2014

Copyright (C) 2014 by authors and Scientific Research Publishing Inc.

This work is licensed under the Creative Commons Attribution International License (CC BY).

http://creativecommons.org/licenses/by/4.0/

(c) (i) Open Access

\section{Abstract}

Background: The aim of this study is to evaluate the role of IMA and the effect of Type-2 diabetes in peripheral arterial ischemic patient. Methods: In our study, randomized patient groups to be undergone peripheral surgery, consisting of 16 diabetic and 12 non-diabetic patients diagnosed with peripheral arterial disease, have been examined. After standard anesthesia, the surgery was performed by the same surgical team, blood samples were taken before and after cross-clamp, and the levels of ischemia modified albumin (IMA), which is a new ischemic marker, were studied spectrophotometrically. Demographic data were compared with the type-2 diabetes association. Results: In this study, we found that there were no correlation with the ankle brachial index (ABI) and risk factors of diabetes between postoperative IMA levels (P: 0.47). Preoperative IMA serum blood levels were significantly higher in patients with peripheral arterial disease (P: 0.70), and positively associated with $A B I$ (diabetic group P: 0.04, non-diabetic group P: 00). Also, no correlation was found with $X$ clamp times, ABI, and IMA blood levels (P: 0.30). In diabetic group, ischemia marker increase was high and there was a positive correlation between HbA1c levels (P: 0.002). Conclusion: Our study suggested that, preoperative serum IMA levels were positively associated with ABI. In diabetic group, the increase in IMA levels was high and there was positive correlation between HbA1c levels. IMA was a benefit marker for monitoring and early diagnosis of the peripheral arterial disease.

${ }^{*}$ Corresponding author.

How to cite this paper: Gencpinar, T., Ayoğlu, U., Aykut, K., Albayrak, G., Ellidag, H.Y., Yılmaz, M., Sağdıc, K. and Emmiler, M. (2014) Effect of Peripheral Arterial Disease on Serum Ischemia-Modified Albumin Levels in Type 2 Diabetic Patients. World Journal of Cardiovascular Surgery, 4, 81-86. http://dx.doi.org/10.4236/wjcs.2014.45013 


\section{Keywords}

\section{Artery, Diabetes Mellitus, Ischemia, IMA}

\section{Introduction}

Peripheral arterial disease (PAD) is an important manifestation of atherosclerosis increasing the cardiovascular mortality and morbidity. Early diagnosis of PAD can reduce the progression of the disease. It can be increased most commonly by smoking, hypertension (HT), diabetes, kidney and heart failure disease, and coronary artery disease (CAD). Age, hyper cholesterol, alcohol, high systolic blood pressure, and hyperhomocysteine are great risk factors for the PAD. More than 95\% of the persons with PAD have major cardiovascular risk factors [1]. The population of diabetic patients increases worldwide. Diabetic patients have a high risk of vascular events [1] [2]. The PAD is one of the main factors for diabetic foot ulcers and lower limb amputations. The recovery period of the PAD is important for limb salvage and the quality of life. In the PAD, biomarkers are released from lower limb vascular endothelium into the circulation [2]. New blood tests can measure these biomarkers that recognize and affect the treatment. Biomarkers are also useful for prognosis [2] [3]. Ischemia modified albumin (IMA) is a beneficial ischemia marker but its role in type-2 diabetes mellitus with peripheral arterial disease has not been identified yet. There are few studies in the literature evaluating the ischemia modified albumin levels in patients with PAD. We want to show that IMA serum levels might be affected by ischemia during the PAD. In a period of one year, twenty eight PAD patients were treated by arterial re-vascularization of standard based surgical technique. The ankle brachial index (ABI) is a simple test of diagnosing PAD. The ABI was measured preand post-operative period by using the standard protocol and compared with IMA levels. The IMA levels and clinical parameters were measured. The demographic data were examined. In this study, we examined the clinical importance of diabetic and non-diabetic patients with critical limb ischemia and extended ischemic wounds, compared to a new ischemia marker "IMA" levels.

\section{Materials and Methods}

In our study, randomized patient groups to be undergone peripheral surgery, consisting of 16 diabetic and 12 non-diabetic patients diagnosed with peripheral arterial disease, have been examined. After standard anesthesia, the surgery was performed by the same surgical team, blood samples were taken before and after cross-clamp, and the levels of ischemia modified albumin (IMA), which is a new ischemic marker, were studied spectrophotometrically. Demographic datas were compared with the type-2 diabetes association.

All surgical procedures were performed with standard vascular revascularization technigue and endoscopic techniques were not used. Color Doppler Ultrasound was used before the operations for selection grafts. The saphenous vein was not suitable for grafting in 3 patient's leg due to Doppler examination. The grafts were harvested with scissors and hemoclips used for bleeding. The grafts protected inside of Papaverine $(1.0 \mathrm{mmol} / \mathrm{L})$ mixed with heparinized blood. Also heparinized blood was injected intraluminally into SVG grafts after harvesting. We aimed graft spasm prophylaxis to provide conduits.

All analysis was performed with "Statistical Package For Social Sciences" (SPSS-15 Chicago, IL, USA) and Software Excel (Microsoft-USA). Continuous variables were expressed as mean and standard deviation. The approprity to normal expedition had been checked. Mann-Whitney test was used to determine independent predictors. If the p-value was $<0.05$, the results were considered significant.

\section{Patients}

\subsection{Exclusion Criteria}

Subjects having at least one of those following conditions were excluded.

1). Serum albumin concentrations under $2 \mathrm{~g} / \mathrm{dl}$ and over $5.5 \mathrm{~g} / \mathrm{dl}$.

2). Ischemic situations such as stroke, transient ischemic attack, acute coronary syndrome (ACS) or muscle disease, trauma, shock, malnutrition, pregnancy, liver diseases, dialysis dependent renal failure, neoplasia. 


\subsection{Samples}

Time sequences for blood sample collection:

T1 samples: After anesthesia, before X clamp.

T2 samples: After anesthesia, after X clamp.

Plasma samples were taken into $8 \mathrm{cc}$ gel separator tubes is used to measure IMA. Blood samples were centrifuged after 10 minutes and plasma examination was performed at $20^{\circ} \mathrm{C}$.

\subsection{Measurement of Ischemia-Modified Albumin}

Reduced cobalt to albumin binding capacity (IMA level) was measured by using rapid and colorimetric method developed by Bar-Or et al. [2] [4]. Briefly, $200 \mu \mathrm{L}$ patient serum was transferred into glass tubes and $50 \mu \mathrm{L} 0.1 \%$ $\mathrm{CoCl}_{2}{ }^{*} 6 \mathrm{H}_{2} \mathrm{O}$ (lot S38901-248, Sigma-Aldrich, St. Louis, MO, USA) was added. After gentle shaking, the mixture was incubated for 10 minutes to ensure sufficient cobalt albumin binding. Then, $50 \mu \mathrm{L} 1.5 \mathrm{mg} / \mathrm{mL}$ dithiothreitol (DTT) (lot D5545-1G Sigma-Aldrich) was added as a coloring agent. After 2 minutes, $1 \mathrm{~mL} 0.9 \% \mathrm{NaCl}$ was added to halt the binding between cobalt and albumin. A blank was prepared for every specimen: at the DTT-addition step, $50 \mu \mathrm{L}$ distilled water was used instead of $50 \mu \mathrm{L} 1.5 \mathrm{mg} / \mathrm{mL}$ DTT to obtain a blank without DTT. The absorbance was recorded at $470 \mathrm{~nm}$ with a spectrophotometer (UV1201, Shimadzu, Japan). Color formation in specimens with DTT was compared with color formation in the blank tubes, and the results are expressed as absorbance units (ABSU).

\section{Statistical Analyses}

Direction and significance of the association between non-parametric variables were evaluated by using Chi-square and Fisher's exact test. The significance of the differences in parametric variables between groups was determined by the Mann-Whitney U-test. Spearman's correlation coefficient was used to test the strength of any associations between different variables. A p-value less than 0,05 was accepted as the significance level. In all calculations and statistical analyses, "Statistical Package for Social Sciences" (SPSS-Chicago, IL, USA) 15 and Software Excel (Microsoft-USA) programmed were used.

\section{Results}

Demographic and clinical data of patients are shown in Table 1. There were no significant differences between two groups in terms of gender, age, HT, chronic obstructive pulmonary disease (COPD), and CAD (Table 2). In this study, we found that there was no correlation with the ABI and risk factors of diabetes between postoperative IMA levels (P: 0.4736) (Figure 1). Preoperative IMA median (IQR) (ABSU) serum blood levels were significantly higher in patients with peripheral arterial disease (P: 0.7) (Figure 2). Total cholesterol, low density lipoprotein, triglyceride, HbAlc, albumin serum levels were not different between in patients with DM and non-diabetic group. Preoperative serum blood IMA levels were positively associated with ABI (diabetic group P: 0.0430, non-diabetic group P: 0.0069). Also, no correlation was found with X clamp times, ABI and IMA blood levels (P: 0.3030). In diabetic group, ischemia marker increase was high and there was a positive correlation between HbA1c levels (P: 0.002).

\section{Discussion}

Peripheral arterial disease (PAD) of the lower extremities represents a spectrum of diseases caused by a large number etiology. Atherosclerosis is still specified as the most common cause of peripheral arterial disease; other diseases can reduce limb perfusion. Congenital, inflammatory, and fibro-muscular dysplasia, vasculitis, Buerger's disease, vascular entrapment neuropathies may affect the peripheral circulation [4] [5]. To determine the strategy for the diagnosis and treatment of arterial stenosis as well as ultrasonographic and hemodynamic evaluation, invasive angiographic studies can carried out. These audits can be developed based on low-cost, non-invasive ness, rapid, and reliable diagnosis. Early diagnose and treatment in the PAD is a life-saving condition. With an effective multidisciplinary treatment strategy of the $\mathrm{PAH}$, an improvement in the natural history (myocardial infarction, stroke, amputation and death) and the prevention of multi-organ distortion can be possible. Diabetes mellitus is a strong risk factor that contributes to 3-4 times the incidence of the PAH [5]. The IMA is a sensitive biomarker for ischemic events. Serum levels of the IMA increase in many diseases accompanied 
Table 1. Demographic and clinical datas of patients with PAD.

\begin{tabular}{|c|c|c|c|}
\hline Parameter & DM $(n=16)$ & Non-DM $(n=12)$ & $\mathbf{P}$ \\
\hline Age, mean \pm SD & $60.7 \pm 8.1$ & $65.02 \pm 10.1$ & 0.3 \\
\hline Gender & \multicolumn{2}{|c|}{ All men } & 0.57 \\
\hline Smoker, (n), (\%) & $10(35 \%)$ & $8(20.5 \%)$ & 0.23 \\
\hline CAD, (n), (\%) & 7 & 4 & 0.7 \\
\hline HT, (n), (\%) & 11 & 8 & 0.9 \\
\hline COPD, (n), (\%) & 2 & 4 & 0.3 \\
\hline CAD history & 8 & 5 & 0.7 \\
\hline Diabeticfoot & 7 & 0 & $<0.01$ \\
\hline Aspirine & 10 & 8 & 0.4 \\
\hline DMAH & 5 & 3 & 0.9 \\
\hline Clopidogrel & 8 & 6 & 0.9 \\
\hline Femoropopliteal bypass & 7 & 8 & 0.2 \\
\hline Aortobifemoral bypass (ABF) & 4 & 1 & 0.3 \\
\hline İleofemoral bypass & 2 & - & - \\
\hline Pseudoaneurisym & 2 & - & - \\
\hline Redo-femoropopliteal bypass & 1 & - & - \\
\hline Distallimb bypass & - & 1 & - \\
\hline Redo ABF & - & 1 & - \\
\hline Embolectomy & - & 1 & - \\
\hline Preop-ABI & $0.6(0.52-0.8)$ & $0.5(0.44-0.82)$ & 0.7 \\
\hline Postop-ABI & $0.6(0.53-0.81)$ & $0.6(0.5-0.84)$ & 0.8 \\
\hline$X$ clamptimes. median, IQR & $40(25-45)$ & $42.5(25-52.5)$ & 0.5 \\
\hline Postoperativepulses & & able & \\
\hline
\end{tabular}

Table 2. Laboratory datas.

\begin{tabular}{cccc}
\hline Parameter & DM (n= 16) & Non-DM (n= 12) & P \\
\hline T.kolesterolmedian (IQR) (mg/d) & $168(127-210)$ & $175(137-232)$ & 0.4 \\
Trigliseridemedian (IQR) (mg/dl) & $117(84-177)$ & $136(94-215)$ & 0.6 \\
LDL median (IQR) (mg/dl) & $104(66-143)$ & $101(87-155)$ & 0.5 \\
HbA1c median (IQR) (\%) & $7.3(6.8-8.3)$ & $5.7(4.5-6.4)$ & 0.002 \\
Albumin, median (IQR) (g/dl) & $3.4(2.7-4.1)$ & $3.7(3-4.2)$ & 0.3 \\
Preop IMA median (IQR) (ABSU) & $0.3(0.28-0.31)$ & $0.3(0.27-0.31)$ & 0.7 \\
Postop IMA median (IQR) (ABSU) & $0.3(0.29-0.31)$ & $0.3(0.3-0.31)$ & 0.4 \\
\hline
\end{tabular}

by ischemia. Ischemia-modified albumin is a new biomarker for the identification of tissue hypoxia in acute myocardial and mesenteric-renal ischemia and other non-cardiac conditions such as obesity, polycystic ovary syndrome with or without insulin resistance, normal and complicated pregnancies, hypoxic ischemic brain injury and diabetes mellitus [4] [6]. It is well known that the IMA increases within minutes from the onset of the ischemic event and remains elevated for several hours after the constitution of the tissue hypoxia [6]. The pathophysiological events of ischemia, including hypoxia and free oxygen radicals, result in a conformational change of the N-terminus of albumin, and this new molecule is called as ischemia-modified albumin [6] [7]. Increased plasma IMA levels are used for diagnosing the disease as an early sign for myocardial ischemia. Despite there are a lot of studies about the IMA sensitivity in coronary syndrome, the studies about the role of IMA are quite inadequate in peripheral arterial disease (PAD) surgery. 


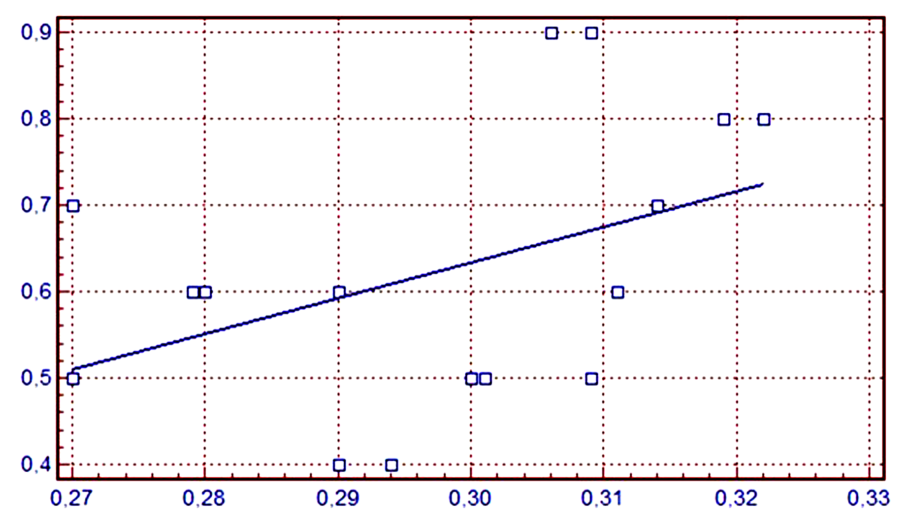

Figure 1. Post-op ABI and IMA levels of diabetic patients.

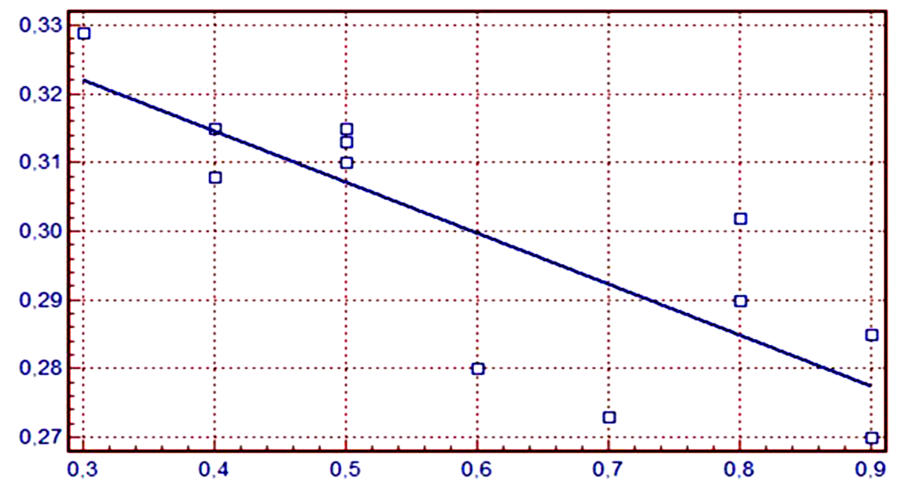

Figure 2. Preop-ABI and IMA levels of non-diabetic patients.

In this study, we plan to investigate the role of new ischemia marker "IMA" and the role of type-2 diabetes in peripheral arterial disease. In our study, 16 diabetic and 12 non-diabetic patients undergone a peripheral bypass surgery with the diagnosis of peripheral arterial disease were randomized. Following the standard procedure of anesthesia in operations implemented by the same surgical team. Standard-dose heparinization (5000 IU) was given preoperatively. Standard anticoagulant therapy (acetil-salicilic acid $100 \mathrm{mg} /$ day and clopidogrel 75 $\mathrm{mg} / \mathrm{day}$ per oral) were given to all postoperative patients. Blood samples were collected before and after the cross clamp. The levels of Ischemia-Modified Albumin were studied in spectrophotometric method. IMA levels and demographic data were compared. Given all of these results, we focused on investigating the sensitivity of IMA during perioperative and postoperative period in the PAD and comparing this test with ankle-brachial index. We found that there were no correlations with the ankle brachial index (ABI) and risk factors of diabetes between postoperative IMA levels (P: 0.4736). Preoperative IMA serum blood levels were significantly higher in patients with peripheral arterial disease (P: 0.7). Preoperative serum blood IMA levels were positively associated with ABI (diabetic group P: 0, 0430, non-diabetic group P: 0.0069). It showed that PAD patients in clinical ischemia condition have high IMA levels and low ABI. Also, no correlation was found with X-clamp times, $\mathrm{ABI}$, and IMA blood levels (P: 0.3030). In diabetic group, the ischemia marker increase was high, and there were positively correlations between HbA1c levels (P: 0.002). High IMA levels in diabetic patients might show us potential vascular disease. In addition, elevation of IMA levels might be related to the severity of PAD.

Cellular stress factors, free oxygen radicals, and high levels of lactic acid are other additional factors influencing the IMA levels [8]. Probably in a very near future, a "multi-marker strategy" will be accepted including some combinations of inflammatory markers like C-reactive protein (CRP), paraoxonase, total oxidant status (TOS) biomarkers in early diagnosis and treatment protocols of ischemia. Among all these studies, the marker mostly gaining importance is the ischemia modified albumin, and is approved by FDA [9] [10]. The consequences are financially beneficial. Successful results and healing in the patient's prognosis will increase the need for such kind of innovative protocols. After PAD operations, the IMA levels have been increased. But in order to measure them in daily routines, it hasn't been suggested in long-lasting follows nowadays. 


\section{Limitations of Ischemia Modified Albumin}

As previously described, the IMA may be increased in settings of non-coronary ischemia. Therefore, the IMA may be less useful in patients with chronic ischemic vascular disease and it should not be used in diagnosis alone as a marker. The second limitation is a genetic variation of two amino acids of the NH2 terminus; therefore, false positive results may be seen in the clinical practice [8] [10]. The frequency of this deletion in the general population is unknown.

\section{Conclusion}

Future studies can increase the identification of unrecognized PADs. New biomarkers such as IMA levels would give advantages to the patients in terms of better compliance and thus avoid major limb amputation.

\section{Disclosure}

The authors declare no conflict of interest.

\section{References}

[1] Hirsch, A.T., Haskal, Z.J., Hertzer, N.R., Bakal, C.W., Creager, M.A., Halperin, J.L., et al. (2006) ACC/AHA Guidelines for the Management of Patients with Peripheral Arterial Disease (Lower Extremity, Renal, Mesentery, and Abdominal Aortic). Circulation, 113, 1474-1547; and Journal of the American College of Cardiology, 47, 1239-1312.

[2] Bar-Or, D., Lau, E. and Winkler, J.V. (2000) A Novel Assay for Cobalt-Albumin Binding and Its Potential as a Marker for Myocardial Ischemia—A Preliminary Report. The Journal of Emergency Medicine, 19, 311-315. http://dx.doi.org/10.1016/S0736-4679(00)00255-9

[3] Norgren, L., Hiatt, W.R., Dormandy, J.A., Nehler, M.R., Harris, K.A., Fowkes, F.G., et al. (2007) Inter-Society Consensus for the Management of Peripheral Arterial Disease (TASC II). Journal of Vascular Surgery, 45, S5-S67. http://dx.doi.org/10.1016/j.jvs.2006.12.037

[4] Kaefer, M., Piva, S.J., De Carvalho, J.A., Da Silva, D.B., Becker, A.M., Coelho, A.C., et al. (2010) Association between Ischemia Modified Albumin, Inflammation and Hyperglycemia in Type 2 Diabetes Mellitus. Clinical Biochemistry, 43, 450-454. http://dx.doi.org/10.1016/j.clinbiochem.2009.11.018

[5] O'Hare, A.M., Katz, R., Shlipak, M.G., Cushman, M. and Newman, A.B. (2006) Mortality and Cardiovascular Risk across the Ankle-Arm Index Spectrum: Results from the Cardiovascular Health Study. Circulation, 113, 388-393. http://dx.doi.org/10.1161/CIRCULATIONAHA.105.570903

[6] Aronow, W.S., Ahn, C., Weiss, M.B. and Babu, S. (2007) Relation of Increased Hemoglobin A (1c) Levels to Severity of Peripheral Arterial Disease in Patients with Diabetes Mellitus. American Journal of Cardiology, 99, 1468-1469. http://dx.doi.org/10.1016/j.amjcard.2006.12.085

[7] Newman, A.B., Siscovick, D.S., Manolio, T.A., Polak, J., Fried, L.P., Borhani, N.O., et al. (1993) Ankle-Arm Index as a Marker of Atherosclerosis in the Cardiovascular Health Study. Circulation, 88, 837-845. http://dx.doi.org/10.1161/01.CIR.88.3.837m

[8] Dahiya, K., Aggarwal, K., Seth, S., Singh, V. and Sharma, T.K. (2010) Type 2 Diabetes Mellitus without Vascular Complications and Ischemia Modified Albumin. Clinical Laboratory, 56, 187-190.

[9] Amstrong, D.G. and Lavery, L.A. (1998) Diabetic Foot Ulcers: Prevention, Diagnosis and Classification. American Family Physician, 57, 1325-1332.

[10] Gençpınar, T., Albayrak, G., Akgol, E., Metin, S.K., Ugurlu, Ş.B. and Hazan, E. (2013) İschemia Modified Albumin Sensitivity of Coronary Artery Bypass Operation. Turkiye Klinikleri Cardiovascular Sciences, 25, 1-7. 